\title{
Interactive tool in gallium arsenide semiconductor modeling: GaAsMod
}

\section{Valentin Loyo-Maldonado, Rene Romero-Troncoso, David Antonio-Torres}

Valentin Loyo-Maldonado, Rene de J. Romero-Troncoso, David AntonioTorres, "Interactive tool in gallium arsenide semiconductor modeling: GaAsMod," Proc. SPIE 3831, Sixth International Conference on Education and Training in Optics and Photonics, (16 June 2000); doi: 10.1117/12.388747

Event: Education and Training in Optics and Photonics (ETOP'99), 1999, Cancun, Mexico 


\title{
An Interactive Tool in Gallium Arsenide Semiconductor Modeling. GaAsMod.
}

\author{
Valentín Loyo-Maldonado ${ }^{a}$, René de J. Romero-Troncoso ${ }^{b}$, David Antonio-Torres ${ }^{a}$ \\ ${ }^{a}$ Centro de Investigación en Ingeniería y Ciencias Aplicadas, \\ Universidad Autonoma del Edo. de Morelos, México. \\ E-mail: vloyo@elec.gla.ac.uk \\ ${ }^{b}$ Facultad de Ingeniería Mecánica Eléctrica y Electrónica, \\ Universidad de Guanajuato, México.
}

\begin{abstract}
In this conference paper we present the development of an interactive tool for gallium arsenide semiconductor modeling. This interactive tool consists in a software package, which is able to model four basic physical phenomena, such as Fermi Level and Mobility, and a few examples of semiconductor devices. We encourage the use of this tool in either, electronic physics lectures or photonics lectures in order to help the complete understanding of these phenomena and devices. GaAsMod can be used to teach in engineering levels as well as in the associate engineering degrees (ISCED5).
\end{abstract}

Keywords: Gallium Arsenide models, Engineering education.

\section{INTRODUCTION}

In general, the behavior of solid-state electronics devices is based mainly in four basic physics phenomena: Fermi Level, Mobility, Carrier Concentration and Resistivity. Quantum physics laws, basically the Schrödiger equation, give us a full description of these phenomena. Any analytical attempt to solve the wave equation is impractical and highly complicated for any phenomenon involving more than a single particle. The students of electronics engineering, and the professionals too, find several problems when trying to solve the wave equation for any particular case present in their daily work. Due to this, some researchers have developed specific models based on experimental data. To date, many models have been developed by this mean, more or less accurate with the real behavior. Models are, generally, equations "forced" to fit with experimental data and, therefore, the relationship in most cases is not able to show the physical phenomena.

Gallium arsenide and gallium arsenide based materials are among the most important materials for manufacturing semiconductor devices. Nowadays, GaAs devices are widely used in electric, electronics and photonics systems, and their applications are countless. To understand how a GaAs device works, we need to study the basic physical phenomena ruling the behavior of the electrons and holes in its crystalline structure. Physics of semiconductor devices is normally addressed in most of the electronics engineering curricula but the success of the student, from the understanding point of view, will depend on the approach we take to teach it.

Textbooks related to this area provide a set of graphics that shows the behavior of the physical phenomena as one variable function, or as a family of curves in the best case. However, due to the nature of these phenomena, in most cases the relationship involves more than three variables and these graphics do not have the flexibility for showing the full interaction between all of them. This is a problem when you try to cover enough cases to show the full interaction in a printed way, because the overwhelming amount of space required. The importance of the topic, and the complexity of the models, in addition to the lack of interactive electronics engineering tools in this specific field, have encouraged us to develop a didactic tool capable to cover this lacks.

Gallium Arsenide Modeling (GaAsMod) interactive software tool provides a new vision to study GaAs physics inside the classroom. It shows, in a qualitative way, the behavior of physical phenomena or device under study. GaAsMod does not show neither the equations nor data, but curves that can be modified by the user within a valid range. In order to illustrate the behavior, each option has parameters that can be modified by few keystrokes changing the curve in the screen. The options included in GaAsMod are: Fermi Level, Mobility, Carrier Concentration, Resistivity, GaAs Resistor, p-n Junction, Metal Semiconductor Transistor (MESFET) and MESFET Capacitor. Based in our experience using the "brother" program of GaAsMod, the Silicon Semiconductor Modeling (SSMod) [1], we reckon that GaAsMod interactive software tool will help electronics engineering students to better understand physical phenomena and basic devices in electronics. Finally, it is convenient to mention that our aim is not to provide specific data or quantities (even when the software can do it), but show behaviors and trends with the help of high quality graphics. In order to make accessible GaAsMod to most of the students, the environment is a stand-alone system for PC's. 


\section{THE MODELS}

The models used to predict the behavior of the different phenomena and devices are, in most cases, complex equations. These equations are no more than mathematical expressions forced to fit experimental data and, therefore, it is difficult to predict from them, how our parameter under study is going to behave. To create models from experimental data is a common task and it can be easily found in scientific literature. Different models of the same parameter are created for different environmental conditions, different factors affecting it and even for different geometries. To choose the right model for one parameter is some times non trivial and will depend on our specific preferences or requirements. In order to develop GaAsMod, several models were used. The models were consistent with experimental data and valid within the whole range of simulations we present.

2.1. Fermi Level. Fermi Level $E_{F}$ is the probability for an energy level to be occupied by an electron. It determines semiconductor type, doping concentration and carrier distribution. For GaAs, the bandgap $E g$ is around $1.44 \mathrm{eV}$, this means that the difference between the conduction band $E_{C}$ and the valence band $E_{V}$ is $1.44 \mathrm{eV}$. For an intrinsic semiconductor, the Fermi Level lies very close to the middle of the bandgap. When impurities are introduced into the sample, the Fermi Level closes to one of the bands, for donor impurities, it closes to $E_{C}$, and for acceptor impurities, it closes to $E_{V}$. The variables involved in Fermi Level model are temperature $T$ and impurity concentration $N_{B}$. The typical GaAsMod screen for this option is shown in figure 2. The model implemented in the program was reported by [2]

$$
E_{F}=\frac{1}{2}\left(E_{c}+E_{d}\right)+\frac{k T}{2} \ln \left(\frac{N_{D}}{2 U_{c}}\right)-k T \sinh ^{-1}\left(\sqrt{\left.\frac{U_{c}}{8 N_{D}} e^{-\left(E_{c}-E_{D} / 2 k T\right.}\right)}\right)
$$

where $N_{D}$ is the tellurium impurity density,

$k$ is the Boltzmann constant and

$U_{C}$ is the effective state density for electrons, and is given by:

$$
U_{c}=2\left(\frac{2 \pi m_{n}^{*} k T}{h^{2}}\right)^{3 / 2}
$$

2.2. Mobility. The change in the drift velocity $v_{d}$ related to the electric field $E$ is known as mobility $\mu$. This variable is one of the most important in the study of semiconductor behavior and it is present in practically all the models of electronic devices. An n-type device is faster than a p-type device because electron mobility $\mu_{n}$ is higher than hole mobility $\mu_{p}$. Throughout the lattice, a carrier suffers several interactions modifying its trajectory due to scattering mechanisms. Among the most important mechanisms are: lattice scattering, ionized impurities scattering and drift speed saturation.

2.2.1. Lattice Scattering. This model was developed by Blakemore in order to model the behavior of the mobility with respect to temperature [3]:

$$
\begin{aligned}
& \mu_{\mathrm{n}}=8000\left(\frac{300}{\mathrm{~T}}\right)^{2.3} \\
& \mu_{\mathrm{p}}=380\left(\frac{300}{\mathrm{~T}}\right)^{2.3}
\end{aligned}
$$

where $\mu_{n}$ and $\mu_{p}$ are given in $\mathrm{cm}^{2} / \mathrm{V}^{*}$ seg and $T$ is the temperature in ${ }^{\circ} \mathrm{K}$. The lattice scattering screen for n-type mobility is shown in figure 3.

2.2.2. Ionized Impurities Scattering. This model was proposed by Caughey and Thomas [4] to model the behavior in silicon, but by changing the corresponding weights of the asymptotic mobility at high impurities concentration [5], it is also applicable to gallium arsenide; the model and the parameters for GaAs are given next: 


$$
\mu_{\mathrm{In}, \mathrm{p}}=\mu_{\min (\mathrm{n}, \mathrm{p})}+\frac{\mu_{\mathrm{Ln}, \mathrm{p}}-\mu_{\min (\mathrm{n}, \mathrm{p})}}{1+\left(\frac{\mathrm{N}}{\mathrm{N}_{\mathrm{ref}(\mathrm{n}, \mathrm{p})}}\right)^{\alpha \mathrm{n}, \mathrm{p}}}
$$

\begin{tabular}{|c|c|c|}
\hline & n-type & p-type \\
\hline$\mu_{\min }\left(\mathrm{cm}^{2} / \mathrm{V}^{*} \mathrm{seg}\right)$ & 1500 & 50 \\
\hline $\mathrm{N}_{\mathrm{ref}}\left(\mathrm{cm}^{-3}\right)$ & $10^{17}$ & $3.232 \times 10^{17}$ \\
\hline$\alpha$ & 0.5 & 0.4956 \\
\hline
\end{tabular}

The lattice and ionized impurities scattering screen for n-type mobility is shown in Figure 4.

2.2.3. Saturation of the Drift Velocity. This model is proposed in [6] and exposed below.

For the electron case,

$$
\begin{aligned}
& \mu_{n}^{\mathrm{LIF}}=\frac{\mu_{\mathrm{n}}^{\mathrm{LI}}\left[1+\mathrm{A}\left(\mu_{\mathrm{n}}^{\mathrm{LI}} \frac{\mathrm{F}_{\mathrm{n}}}{\mathrm{V}_{\mathrm{n}}^{\text {sat }}}\right)^{\mathrm{t}-1}\right]}{1+\mathrm{A}\left(\mu_{\mathrm{n}}^{\mathrm{LI}} \frac{\mathrm{F}_{\mathrm{n}}}{\mathrm{V}_{\mathrm{n}}^{\text {sat }}}\right)} \\
& \mathrm{V}_{\mathrm{n}}^{\text {sat }}=6 \times 10^{6} \frac{\mathrm{cm}}{\mathrm{s}}\left[\left(1+\frac{\mu_{\mathrm{n}}^{\mathrm{LI}}}{10^{4} \frac{\mathrm{cm}^{2}}{\mathrm{Vs}}}\right)-\left(\frac{\mu_{\mathrm{n}}^{\mathrm{LI}}}{5.477 \times 10^{6} \frac{\mathrm{cm}^{2}}{\mathrm{Vs}}}\right)^{2}\right] \\
& \mathrm{A}=10^{-2}+0.6\left[\exp \left(\frac{\mu_{\mathrm{n}}^{\mathrm{LI}}}{10^{3} \frac{\mathrm{cm}^{2}}{\mathrm{Vs}}}-2\right)+\exp \left(7-\frac{\mu_{\mathrm{n}}^{\mathrm{LI}}}{285.72 \frac{\mathrm{cm}^{2}}{\mathrm{Vs}}}\right)\right]^{-1} \\
& t=4+1280 \operatorname{senh}^{-1}\left(\frac{\mu_{\mathrm{n}}^{\mathrm{LI}}}{250 \frac{\mathrm{cm}^{2}}{\mathrm{Vs}}}\right)
\end{aligned}
$$

and for hole case,

$$
\begin{gathered}
\mu_{\mathrm{p}}^{\mathrm{LIF}}=\frac{\mu_{\mathrm{p}}^{\mathrm{LI}}}{1+\frac{\mu_{\mathrm{p}}^{\mathrm{LI}} \mathrm{F}_{\mathrm{p}}}{\mathrm{V}_{\mathrm{p}}^{\text {sat }}}} \\
\mathrm{V}_{\mathrm{p}}^{\text {sat }}=1.5 \times 10^{7} \frac{\mathrm{cm}}{\mathrm{s}}
\end{gathered}
$$

where $F n$ and $F p$ represent the applied electric fields.

2.3. Carrier Concentration. A carrier available for conduction in a Semiconductor sample is known as free carrier. The concentration of this carrier is deter0mined by the amount of active impurities present in the lattice. Among other parameters, 
free carrier concentration determines mobility and resistivity. The typical GaAsMod screen for this option is shown in figure 5. Carrier concentration $n$ depends on Fermi Level $E_{F}$ and temperature $T$. From the Maxwell distribution and by Boltzmann approximation the following model it can be obtained.

$$
n=U_{c} e^{-\left(E_{c}-E_{F} / k T\right)}
$$

2.4. Resistivity. The flow capacity of carriers under an applied electric field is known as conductivity $\sigma$, its reciprocal is resistivity $\rho$ and determines the electric characteristics on devices. The typical GaAsMod screen for this option is shown in figure 6. Resistivity depends on mobility $\mu$ and carrier concentration $n$ and it is defined by:

$$
\rho=\frac{1}{\sigma}=\frac{1}{q\left(\mu_{n} n+\mu_{p} p\right)}
$$

2.5. Galliun Arsenide Resistor. A doped GaAs sample acts like a resistor. The resistor value will be determined by dimensions and doping (type of impurities and concentration). These physical parameters modify the resistivity of the sample and it depends on temperature, mobility and carrier concentration as seen in the previous section. In order to know the resistor final value, we must consider the resistivity and cross section area values as shown.

$$
R=\rho A
$$

where: $R$ is the resistance of the sample,

$\rho$ is the resistivity and

$A$ is the cross section area.

2.6. p-n Junction. When we join a p-type material with an $n$-type material, the result is a p-n junction or diode. The parameters that characterize this device are threshold voltage and its driven current capability. These parameters are affected mainly by doping. Diodes are very sensitive to temperature variations. The typical GaAsMod screen for this option is shown in Figure 7.

In order to calculate the I-V curves, we use

$$
J=J_{S}\left(e^{q v / k T}-1\right)
$$

where: $J s$ is the saturation current density

2.7. MESFET Capacitor. Internal device capacitances in GaAs MESFETs play an important role in determining the performance of GaAs devices and integrated circuits. In the simplest way, the devices capacitances are modeled as capacitances of Schottky diodes connected between the gate and the source, and the gate and the drain, but this model does not take into account the dependence of those capacitances on the variation of the drain-gate voltage; a model that incorporates this dependence is developed in [7] and shown below:

$$
\mathrm{C}_{\mathrm{gs}}=\mathrm{C}_{\mathrm{gs} 0}\left(\frac{\mathrm{K}_{2} \mathrm{~K}_{1}}{\left(1-\frac{\mathrm{V}_{\mathrm{n}}}{\mathrm{V}_{\mathrm{bi}}}\right)^{\frac{1}{2}}}+\mathrm{K}_{3}\right)
$$

where $V_{b i}$ is the built-in potential originated by the metal-semiconductor junction, $C_{g s}$ is the drain-gate capacitance at a zerobias voltage, which can be given as a manufacturing parameter or calculated from [8]: 


$$
\mathrm{C}_{\mathrm{gs} 0}=\frac{1}{2}\left(\frac{\varepsilon \mathrm{qN}_{\mathrm{d}}}{2 \mathrm{~V}_{\mathrm{bi}}}\right)^{\frac{1}{2}}
$$

given in $\mathrm{F} / \mathrm{cm}^{2}$, where $\varepsilon$ is the GaAs relative permitivity given in $\mathrm{F} / \mathrm{cm}, q$ is the electron charge in coulombs and $N_{d}$ is the channel doping in $\mathrm{cm}^{-3}$. The remaining variables that model the behavior of $C g s$ with respect to $V g s$ are [7]:

$$
\begin{aligned}
& \mathrm{V}_{\max }=0.5 \\
& \delta=0.2 \\
& \mathrm{~K}_{1}=\frac{1}{2}\left\{1+\frac{\mathrm{V}_{\mathrm{e}}-\mathrm{V}_{\mathrm{TO}}}{\left[\left(\mathrm{V}_{\mathrm{e}}-\mathrm{V}_{\mathrm{TO}}\right)^{2}+2\right]^{\frac{1}{2}}}\right\} \\
& \mathrm{K}_{2}=\frac{1}{2}\left\{1+\frac{\mathrm{V}_{\mathrm{gs}}-\mathrm{V}_{\mathrm{gd}}}{\left[\left(\mathrm{V}_{\mathrm{gs}}-\mathrm{V}_{\mathrm{gd}}\right)^{2}+(\underline{1})^{2}\right]^{\frac{1}{2}}}\right\} \\
& \mathrm{K}_{3}=\frac{1}{2}\left\{1-\frac{\mathrm{V}_{\mathrm{gs}}-\mathrm{V}_{\mathrm{gd}}}{\left[\left(\mathrm{V}_{\mathrm{gs}}-\mathrm{V}_{\mathrm{gd}}\right)^{2}+\left(\frac{1}{}\right)^{2}\right]^{\frac{1}{2}}}\right\} \\
& \mathrm{V}_{\mathrm{e}}=\frac{1}{2}\left\{\mathrm{~V}_{\mathrm{gs}}+\mathrm{V}_{\mathrm{gd}}+\left[\left(\mathrm{V}_{\mathrm{gs}}-\mathrm{V}_{\mathrm{gd}}\right)^{2}+\left(\frac{1}{-}\right)^{2}\right]^{\frac{1}{2}}\right\} \\
& \mathrm{V}_{\mathrm{AUX}}=\frac{1}{2}\left\{\mathrm{~V}_{\mathrm{e}}+\mathrm{V}_{\mathrm{TO}}+\left[\left(\mathrm{V}_{\mathrm{e}}-\mathrm{V}_{\mathrm{TO}}\right)^{2}+2\right]^{\frac{1}{2}}\right\} \\
& \text { IF } \mathrm{V}_{\mathrm{AUX}}<\mathrm{V}_{\max } \text { THEN } \mathrm{V}_{\mathrm{n}}=\mathrm{V}_{\mathrm{AUX}} \\
& \text { ELSE } \quad V_{n}=V_{\max }
\end{aligned}
$$

$V_{T O}$ is the threshold voltage of the MESFET and $V_{g s}$ and $V_{g d}$ are the gate-source and the gate-drain voltages, respectively. The MESFET Capacitor screen is shown in figure 8.

2.8. MESFET. MESFETs are widely used in applications such as amplifiers, microwaves and digital integrated circuits. In such applications, it is very important to know the static behavior of the MESFET, and this can be known through the Current-Voltage characteristic curves. The Current-Voltage behavior can be obtained from the Shockley model [8] developed below for a depletion-mode MESFET. The threshold voltage can be determined by

$$
\mathrm{V}_{\mathrm{T}}=-\mathrm{V}_{\mathrm{po}}+\mathrm{V}_{\mathrm{bi}}
$$

where $V_{p o}$ is the pinch-off voltage of the channel given by 


$$
\mathrm{V}_{\mathrm{po}}=\frac{\mathrm{qN}_{\mathrm{d}} \mathrm{A}^{2}}{2 \varepsilon}
$$

where $V_{b i}$ is the built-in potential, $q$ is the electron charge in coulombs, $N_{d}$ is the channel doping in $\mathrm{cm}^{-3}, A$ is the channel thickness width in $\mathrm{cm}$ and $\varepsilon$ is the GaAs permitivity given in $\mathrm{F} / \mathrm{cm}$.

The Current-Voltage behavior presents two operating regions: linear and saturation, modeled in the following way:

i) If $\mathrm{V}_{\mathrm{ds}}<\mathrm{V}_{\mathrm{gs}}-\mathrm{V}_{\mathrm{T}}$ (linear region)

$$
I_{d s}=g_{0}\left\{V_{d s}-\frac{2}{3} \frac{\left[\left(V_{d s}+V_{b i}-V_{g s}\right)^{\frac{3}{2}}-\left(V_{b i}-V_{g s}\right)^{\frac{3}{2}}\right]}{V_{p o}^{\frac{1}{2}}}\right\}
$$

ii) $\mathrm{Si}_{\mathrm{ds}}>\mathrm{V}_{\mathrm{gs}}-\mathrm{V}_{\mathrm{T}}$ (saturation region)

$$
\left(I_{d s}\right)_{s a t}=g_{0}\left[\frac{1}{3} V_{p o}+\frac{2}{3} \frac{\left(V_{b i}-V_{g s}\right)^{\frac{3}{2}}}{V_{p o}^{\frac{1}{2}}}-V_{b i}+V_{g s}\right]
$$

where in both regions $g_{0}$ represents the conductance of the metallurgical channel, given as

$$
\mathrm{g}_{0}=\left(\mathrm{q} \mu \mathrm{N}_{\mathrm{d}} \mathrm{A}\right) \frac{\mathrm{W}}{\mathrm{L}}
$$

where $\mu$ is the mobility given in $\mathrm{cm}^{2} / \mathrm{V}^{*}$ seg and $W$ and $L$ are the width and the length of the channel, respectively. The MESFET Screen is shown in figure 9.

\section{PROGRAM DESCRIPTION}

GaAsMod was thought from the student point of view. It has to be user friendly and very easy to understand. As soon as the GaAsMod is loaded, the main menu appears together with the instructions bar (figure 1). From here, the student can choose either, the physical phenomenon or the device to study. By selecting the options, the student can then "jump" between the different screens contained in GaAsMod. The parameters affecting one specific device can be changed using the arrows, and the curve on that screen will change automatically. The parameters can only be adjusted to fit the range where the model is valid and therefore, GaAsMod does not show curves which are not backed up with a valid model. With GaAsMod, the student can understand in a qualitative way how certain physical phenomena or device behaves under different temperature, doping conditions, size, etc. GaAsMod holds a total of 17 screens and some of them can be seen in point 7 of this paper. Due to the simplicity of the software, it can be used in early undergraduate curses or, as we are encouraging, in the associated engineering degrees (ISCED5)

\section{REQUIREMENTS}

The CPU requirements are fulfilled for today's PC's architectures. GaAsMod needs at least, a $386 \mathrm{MHz}$ processor with math co-processor, $512 \mathrm{~kb}$ RAM, MS-DOS 3.1, SVGA color monitor and $150 \mathrm{~kb}$ hard disk drive space (it can also be loaded from the floppy drive). The program was developed under Borland C v. 2.0.

\section{CONCLUSIONS}

We have presented a new tool that can be used as an auxiliary when teaching physics of semiconductor devices. GaAsMod consists of interactive curves that change depending on the parameters that are being modified by the user. The small size of the program and the few CPU requirements, make GaAsMod the ideal software to take home by giving the student the option to reinforce their knowledge without the extra laboratory hours. 


\section{REFERENCES}

1. V. Loyo-Maldonado and R. de J. Romero-Troncoso, "Interactive tool in silicon semiconductor modeling: SSMod," Comput Appl Eng Educ, vol. 6, pp. 59-65, 1998.

2. S. Grove, Physics and Technology of Semiconductor Devices, Wiley, New York, 1967.

3. J. S. Blakemore, "Semiconducting and other major properties of Gallium Arsenide", J. Appl. Phys., Vol. 53, no. 10, pp. 123-181, October 1981.

4. D. M. Caughey and R. E. Thomas, "Carrier mobilities in Silicon Empirically related to Doping and Field", Proc. IEEE, Vol. 52, pp. 2192-2193, 1967.

5. M. Hirose, J. Yoshida, and N. Toyoda, "An improved Two-Dimensional Simulation Model for GaAs Mesfet Applicable to LSI design", IEEE Trans. Computer-Aided Design, Vol. 7, pp. 225-230, May 1988.

6. J. Xu and M. Shur, "Velocity-Field Dependence in GaAs", IEEE Trans. Electron Devices, DE-34, no. 8, pp. 18311832, August 1987.

7. J. Michael Golio Editor, Microwave: MESFETs and HEMTs, Ed. Artech House, 1991, ISBN: 0-89006-426-1.

8. Michael Shur, GaAs Devices and Circuits, Ed. Prenum Press, 1987, ISBN: 0-306-42192-5. 


\section{SELECTED SOFTWARE SCREENS}

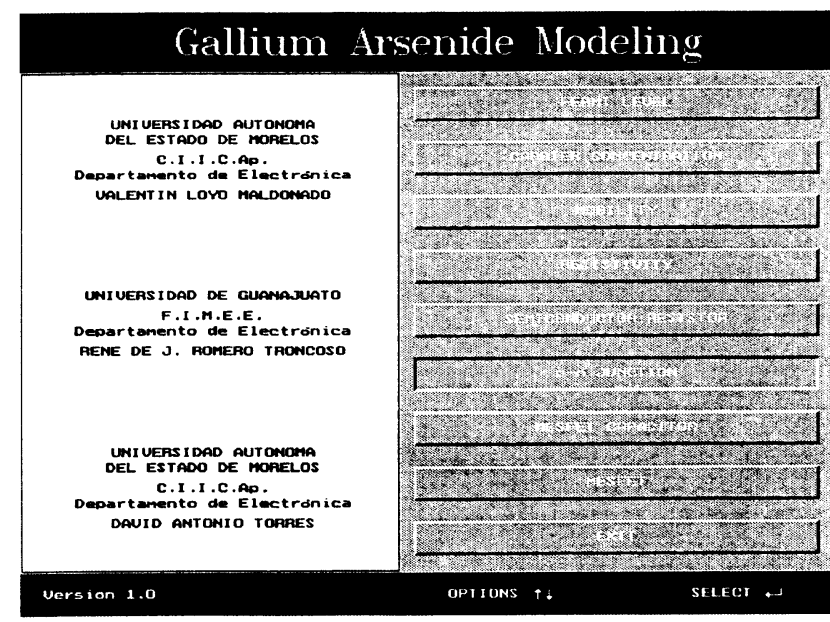

Figure 1. Main Menu.

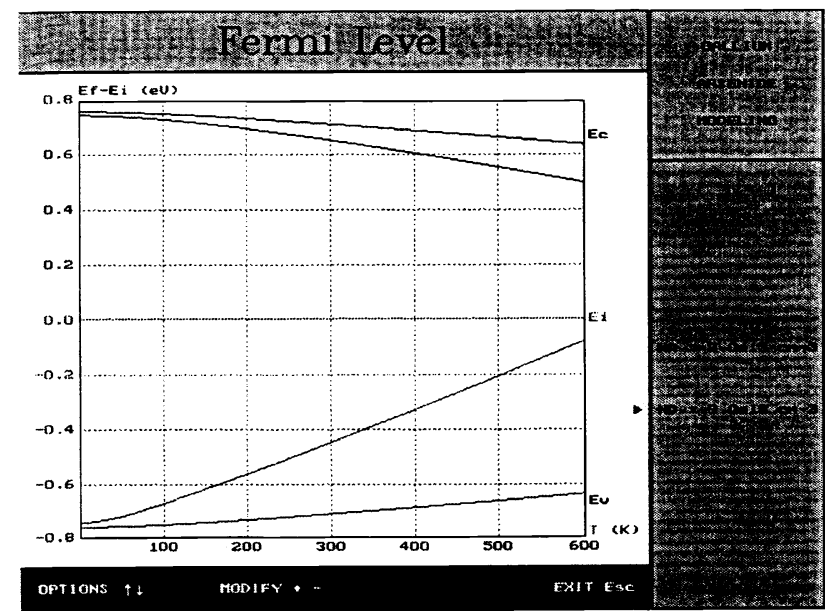

Figure 2. Fermi Level.

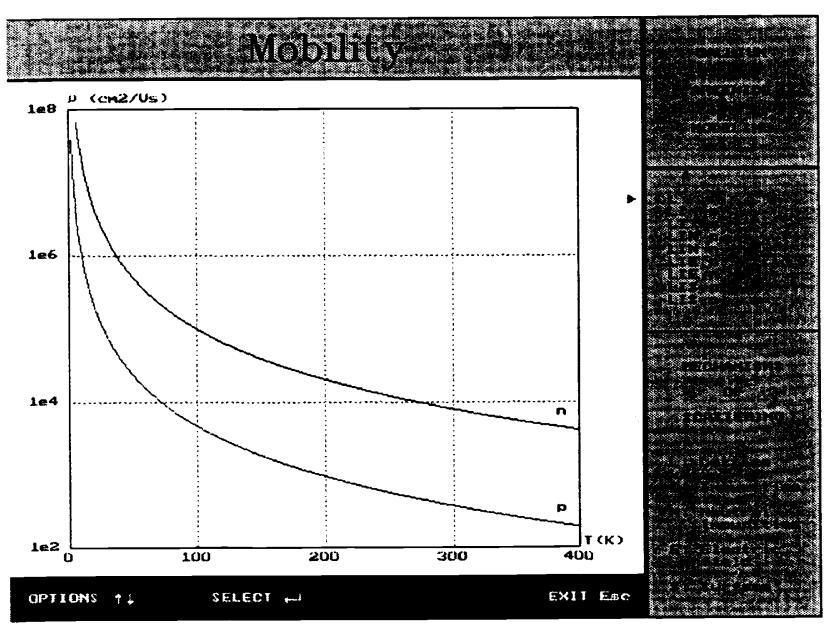

Figure 3. Mobility: Lattice Scattering.

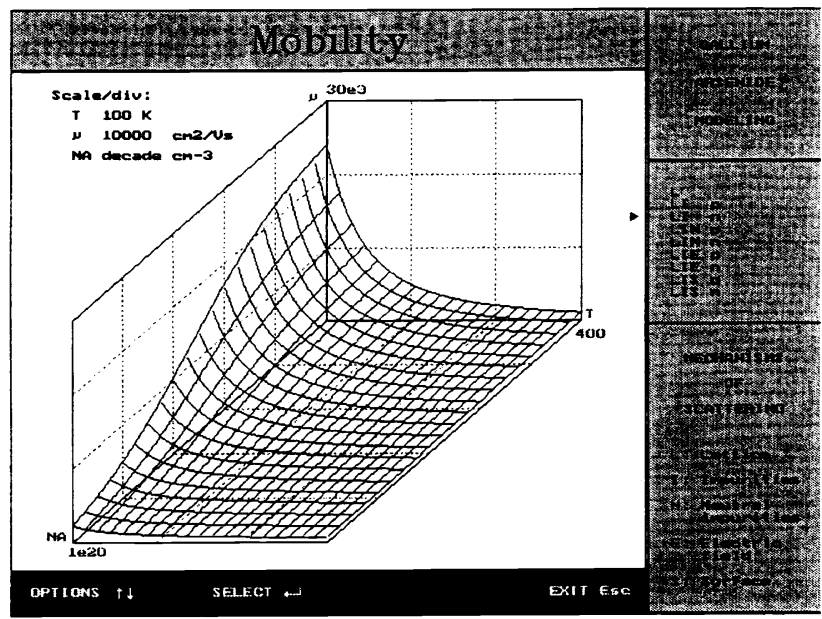

Figure 4. Mobility: n-type Lattice and Impurities Scattering.

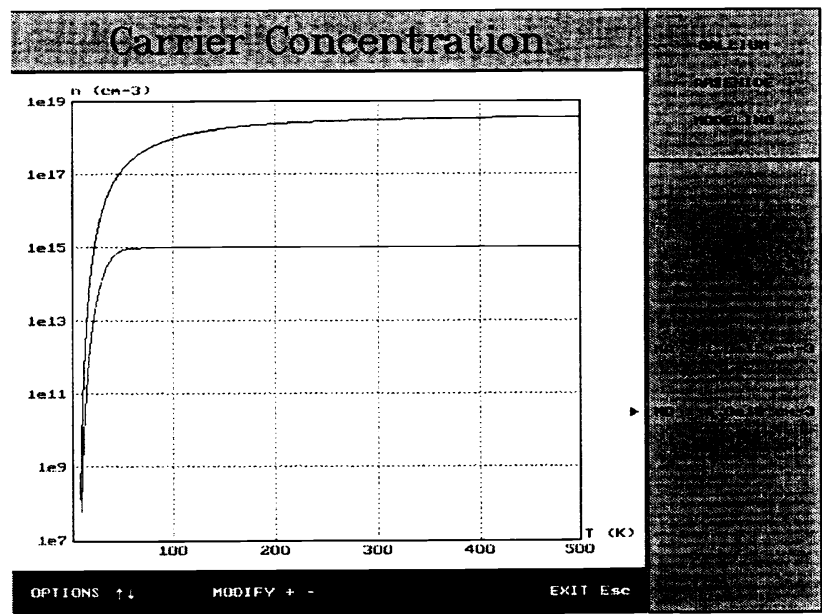

Figure 5. Carrier Concentration.

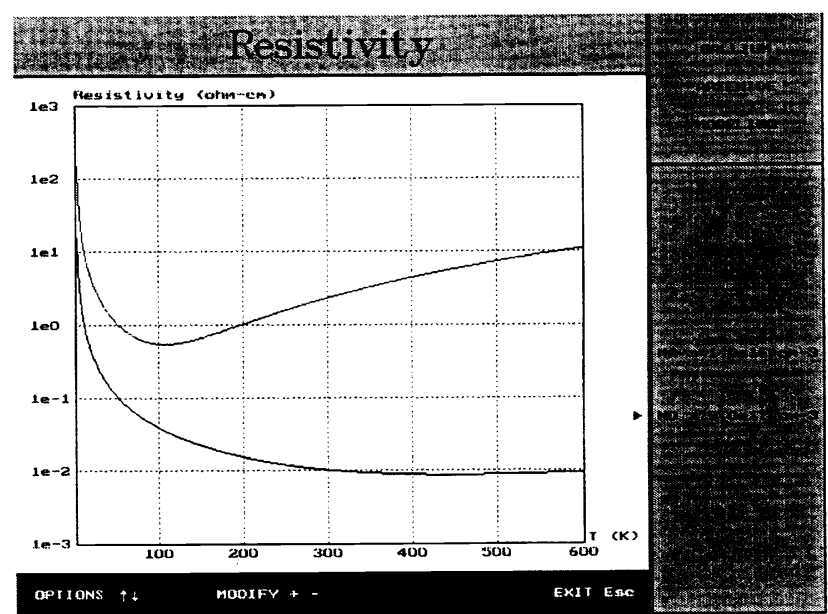

Figure 6. Resistivity. 


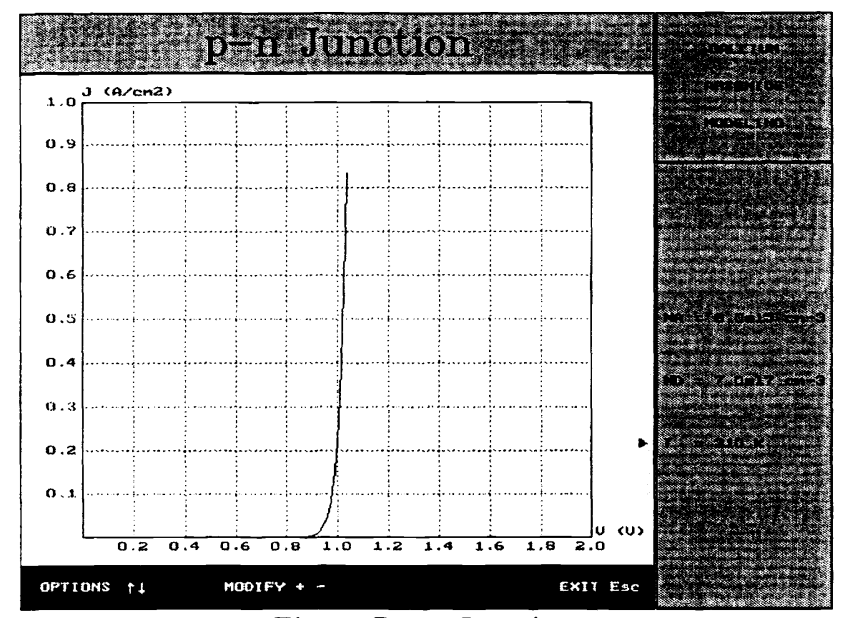

Figure 7. p-n Junction.

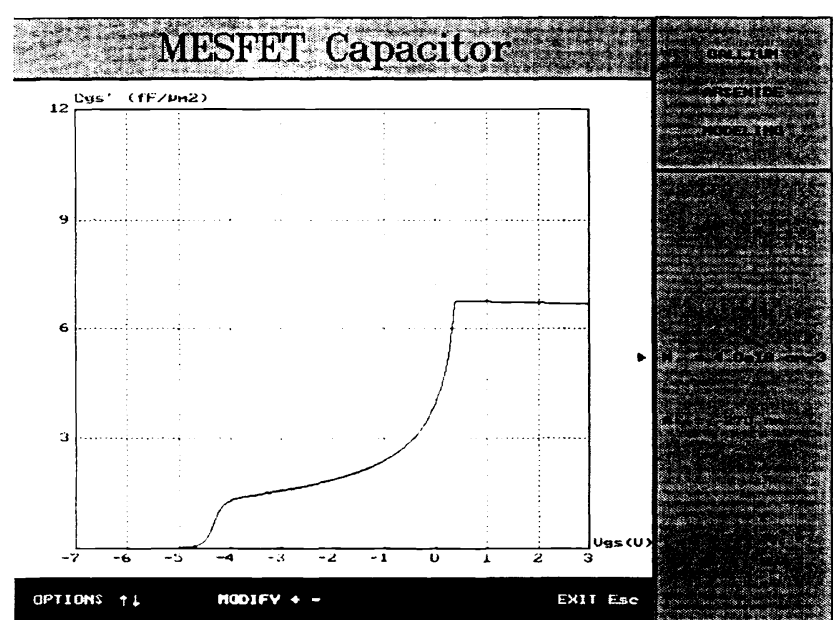

Figure 8. MESFET Capacitor.

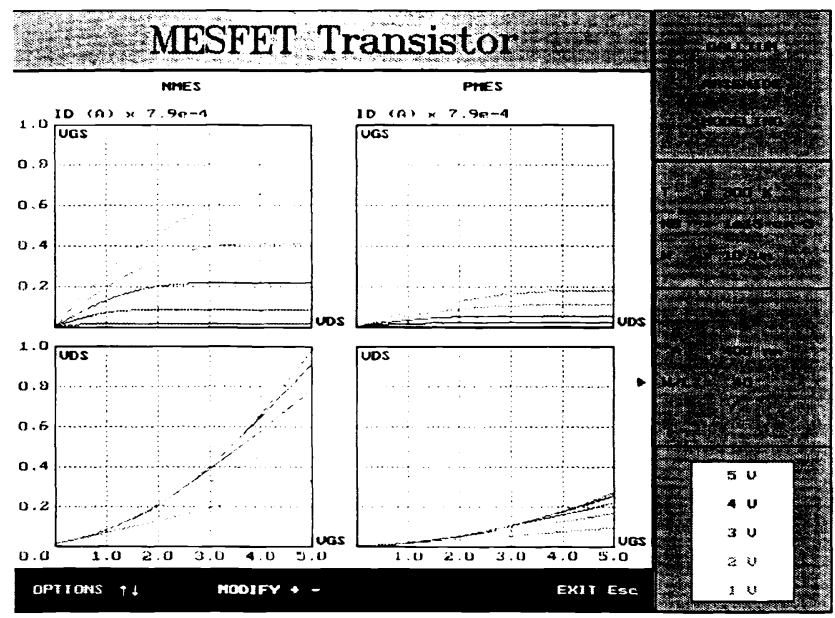

Figure 9. MESFET. 